\title{
PARAMETERS FOR QUANTIFYING BEAM HALO
}

\author{
C.K. Allen and T.P. Wangler \\ Los Alamos National Laboratory, Los Alamos, New Mexico 87545
}

\begin{abstract}
Two different parameters for the quantitative description of beam halo are introduced, both based on moments of the particle distribution. One parameter is a measure of spatial halo formation and has been defined previously by Wangler and Crandall [3], termed the profile parameter. The second parameter relies on kinematic invariants to quantify halo formation in phase space; we call it the halo parameter. The profile parameter can be computed from experimental beam profile data. The halo parameter provides a theoretically more complete description of halo in phase space, but is difficult to obtain experimentally.
\end{abstract}

\section{INTRODUCTION}

We have been investigating parameters that provide a quantitative description of halo. We are looking for a parameter that describes halo in a similar vein with the most important figure-of-merit of beam quality, the rms emittance. The parameter should reflect the identifying characteristics of halo and be useful for both theory and experiment.

It is important to have a definition of halo in 1D spatial projections for which experimental measurements are relatively easy to obtain. With this aim, Wangler and Crandall proposed a quantity for characterization of halo, called the beam profile parameter [3]. Through simulation studies, they found that this parameter was indeed a good indicator of the visually observable halo. However, because of the beam's phase-space rotations, the observed halo in 1D projections oscillates. For example, at some locations the halo may project strongly along the spatial coordinate and only weakly along the momentum coordinate, while at others the reverse is true, and the halo can be hidden from the spatial projection. Therefore it is also important to search for another quantification of halo in 2D phase space distributions, one which is insensitive to the beam's phase space rotations.

Our approach is to extend the 1D work to obtain a halo parameter suitable for description of beam halo in 2D phase space. In so doing, one is lead naturally to the moment invariants presented by Dragt [1] and Lysenko [2]. Specifically, these are polynomial functions of the distribution moments, which are invariant whenever all the forces on the beam are linear (including self-forces). These quantities are known as kinematic invariants and are the consequence of the linear forces and symplectic

\footnotetext{
"Supported by US DOE, NNSA and the Office of Nuclear Energy,

† ckallen@lanl.gov
}

structure imposed by Hamilton's equations. Any quantity built from the kinematic invariants would vary only in situations where nonlinear forces were present.

\section{HALO PARAMETERS}

We consider two parameters for quantifying the halo based on moments of the particle distribution. First we discuss the profile parameter $h$ defined by Wangler and Crandall for the 1D spatial projections. We then introduce the halo parameter $H$ for the $2 \mathrm{D}$ phase-space distributions. The halo parameter generalizes the profile parameter using kinematic invariants.

\subsection{Profile Parameter for Continuous Beams}

A general characteristic of beam halo is the increased population of the outer portion of the beam. The profile parameter describes this feature in the spatial coordinate. Let the coordinates of one phase plane be $(q, p)$, where $q$ and $p$ are the spatial and momentum coordinates, respectively. The spatial profile parameter, denoted $h$, is then defined for continuous beams as [3]

$$
h \equiv<q^{4}>/<q^{2}>^{2}-2,
$$

where $\langle\cdot\rangle$ is the moment operator, or average over the particle distribution. Note that $h$ involves only spatial moments of the distribution. The constant in the above definition is chosen to normalize the parameter to the value 0 for a $\mathrm{KV}$ distribution, for which there is no halo. For a Gaussian distribution $h=1$. Multi-particle simulations show that significant halo presence in a 1D projection corresponds to $h>1$.

The definition of $h$ is essentially that of the kurtosis of the beam, where we have used the value 2 instead of 3 in the usual definition. Kurtosis is typically used to compare the "peakedness" of a distribution to that of a Gaussian. Flatter distributions have negative kurtosis while sharper ones have positive kurtosis. With the value 2 , we normalize to a uniform distribution rather than a Gaussian; this seems appropriate for beam dynamics.

\subsection{Halo Parameter for Continuous Beams}

To describe presence of halo in 2D phase space, we introduce the beam halo parameter $H$. If the motion is uncoupled between phase planes, the following quantities are kinematic invariants of motion [1]:

$$
\begin{aligned}
& I_{2} \equiv<q^{2}><p^{2}>-<q p>^{2}, \\
& I_{4} \equiv<q^{4}><p^{4}>+3<q^{2} p^{2}>^{2}-4<q p^{3}><q^{3} p>.
\end{aligned}
$$

Then we define the halo parameter $H$ as 


$$
\begin{aligned}
H & \equiv \sqrt{3 I_{4}} / 2 I_{2}-2, \\
& =\frac{\sqrt{3<q^{4}><p^{4}>+9<q^{2} p^{2}>^{2}-12<q p^{3}><q^{3} p>}}{2<q^{2}><p^{2}>-2<q p>^{2}}-2 .
\end{aligned}
$$

The constants are chosen for normalization consistent with that of the profile parameter. Thus, in situations of elliptical symmetry in phase space, $H$ will have a value 0 for the KV distribution and a value 1 for the Gaussian distribution. Multi-particle simulations show that significant halo in the $2 \mathrm{D}$ phase-space projection corresponds to $H>1$.

\subsection{Parameter Comparison for Beams with Elliptical Symmetry}

For idealized beam distributions with elliptical symmetry in 2D phase space, the projected density function $\rho(q, p)$ has the form

(4) $\quad \rho(q, p)=f\left(\gamma q^{2}+2 \alpha q p+\beta p^{2}\right)$, where $\alpha, \beta, \gamma$ are the Courant-Snyder parameters and $f(\cdot)$ is a real, positive function. The moments of this distribution may be computed analytically in terms of the moments of $f$. For example, we have

$$
\begin{aligned}
& \left\langle q^{4}\right\rangle=\frac{3}{8} \beta^{2} \frac{F_{2}}{F_{0}}, \quad\left\langle q^{2} p^{2}\right\rangle=\left(\frac{3 \alpha^{2}}{8}+\frac{1}{8}\right) \frac{F_{2}}{F_{0}}, \\
& \left\langle p^{4}\right\rangle=\frac{3}{8} \gamma^{2} \frac{F_{2}}{F_{0}}, \quad\left\langle q p^{3}\right\rangle=-\frac{3}{8} \alpha \gamma \frac{F_{2}}{F_{0}},
\end{aligned}
$$

where

$$
F_{0} \equiv \int_{0}^{\infty} f(s) d s, F_{1} \equiv \int_{0}^{\infty} s f(s) d s, F_{2} \equiv \int_{0}^{\infty} s^{2} f(s) d s .
$$

Using these relations, we find the following relationship for the profile and halo parameters

$$
\text { (7) } h=H=\frac{3}{2} \frac{\left(F_{2} / F_{0}\right)}{\left(F_{1} / F_{0}\right)^{2}}-2 \text {. }
$$

Thus, for elliptically symmetric distributions satisfying Eq. (4), the profile and halo parameters are analytically equivalent. However, more general beam distributions (e.g., from multi-particle simulations) are not elliptically symmetric, and thus Eq. (7) is not satisfied.

\subsection{Bunched Beams and 6D Phase Space}

Wangler and Crandall provided a separate normalization constant for bunched beams. The constant $15 / 7$ was chosen so that the profile parameter $h$ would be zero for a uniform density bunch in $x y z$ space. The resulting profile parameter redefinition is

$$
h \equiv<q^{4}>/<q^{2}>^{2}-\frac{15}{7} .
$$

Likewise, for bunched beams we redefine the halo parameter $H$ as

$$
H \equiv \sqrt{3 I_{4}} / 2 I_{2}-\frac{15}{7} .
$$

Note that the bunched-beam constant $15 / 7 \approx 2.143$ is close to the continuous-beam value of 2 .
Next, we investigate the halo parameters for bunched beams with ellipsoidal symmetry. In six-dimensional phase space, the distribution $\rho$ has the form

$$
\rho\left(q_{1}, p_{1}, q_{2}, p_{2}, q_{3}, p_{3}\right)=q f\left(z^{T} Q z\right),
$$

where

$$
z \equiv\left(\begin{array}{llllll}
q_{1} & p_{1} & q_{2} & p_{2} & q_{3} & p_{3}
\end{array}\right)^{T},
$$

$f$ is a positive real function, and $Q$ is some symmetric, positive definite, real $6 \times 6$ matrix (generalized CourantSnyder parameters). For distributions described by Eq. (10), it is again possible to compute analytically the values of the profile and halo parameters, $H$ and $h$. We first compute the invariants $I_{2}$ and $I_{4}$. They are

$$
I_{2}=\frac{1}{36} \frac{F_{3}^{2}}{F_{2}^{2}} \frac{1}{\operatorname{det} Q} \quad \text { and } \quad I_{4}=\frac{1}{192} \frac{F_{4}^{2}}{F_{2}^{2}} \frac{1}{(\operatorname{det} Q)^{2}} .
$$

The halo and profile parameters depend only upon the distribution function $f$

$$
H=h=\frac{9}{4} \frac{F_{4} F_{2}}{F_{3}^{2}}-\frac{15}{7} .
$$

For the case of ellipsoidal symmetry in 6D phase space, we find that both parameters are again equal. Generally however, beam distributions do not have ellipsoidal symmetry and Eq. (13) is not satisfied.

\section{NUMERICAL VALUES}

To illustrate typical behavior of the profile and halo parameters we present some numerical values for particular beam distributions.

\subsection{Parameter Values of Common Distributions}

We tabulate the values of the profile and halo parameters for several standard analytic distributions generally considered not to having significant halo. Table 1 lists the values of $h$ and $H$ both for the continuous and bunched beam case. In the continuous beam case, we assume the distribution is uniform in any $2 \mathrm{D}$ projection (Kapchinskij-Vladmirskij distribution). In the bunched beam case, we assume that the distribution is uniform in any $3 \mathrm{D}$ projection. Notice that all values lie between 0 and 1 , unlike beams from the multi-particle simulations that show strong halo (see below).

Table 1. Parameter values for analytic distributions

\begin{tabular}{|l|c|c|}
\hline \multirow{2}{*}{ Distribution } & \multicolumn{2}{|c|}{$H, h$} \\
\cline { 2 - 3 } & Continuous & Bunched \\
\hline Uniform & 0 & 0 \\
\hline Parabolic & $1 / 4$ & $4 / 21$ \\
\hline Gaussian & 1 & $6 / 7$ \\
\hline Hollow & $1 / 4$ & $75 / 112$ \\
\hline
\end{tabular}




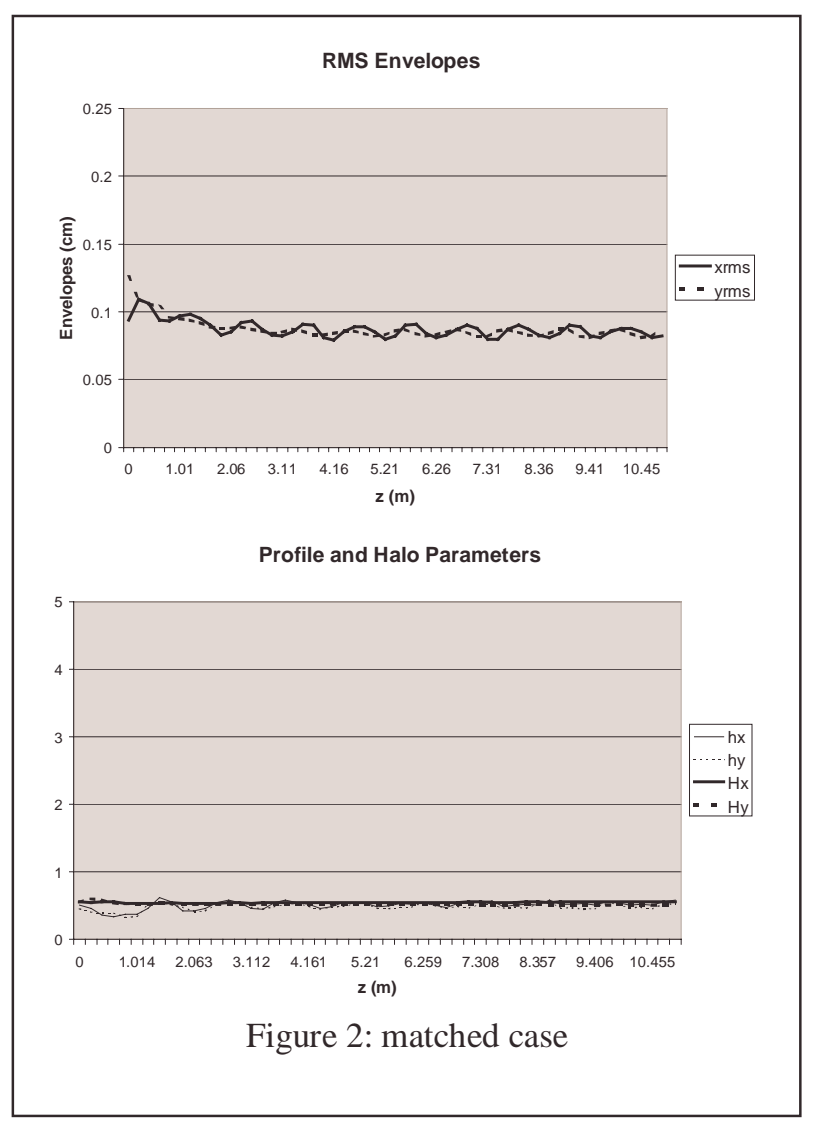

\subsection{Numerical Simulations}

To illustrate the behavior of the halo parameters $h$ and $H$ for distributions in multi-particle simulations, we consider simulations of the halo experiment at the Los Alamos Low-Energy Demonstration Accelerator (LEDA). The Halo Experiment is designed to produce halo by mismatching the beam into a 52-quadrupole periodic FODO lattice. The beam is initially bunched; however, since there is no longitudinal focusing, it debunches as it propagates down the channel. Here we use the continuous beam profile parameter $h$ halo parameter $H$.

We consider two cases, the matched case and a mismatch that excites the quadrupole envelope mode. These cases are shown in Figure 2 and Figure 1, respectively. We see that the halo parameters $H_{x}, H_{y}$ and the profile parameters $h_{x}, h_{y}$ are approximately constant at values less than one for the matched beam. When the beam is mismatched, the $H$ parameters smoothly increase while the $h$ parameters are oscillatory about an increasing mean. Both the $h$ and $H$ halo parameters assume values larger than one, indicating strong halo formation.

Unlike the idealized case of elliptical symmetry where $h=H$, the mismatched beam in Figure 1 show that the parameters can be quite different for the more general distributions. As mentioned above, it has been observed that the halo can "hide" in phase space, so that it is not observed in some spatial projections. The variations of the profile parameter $h$ are reflecting this fact, oscillating about the smoothly varying $H$ parameter.

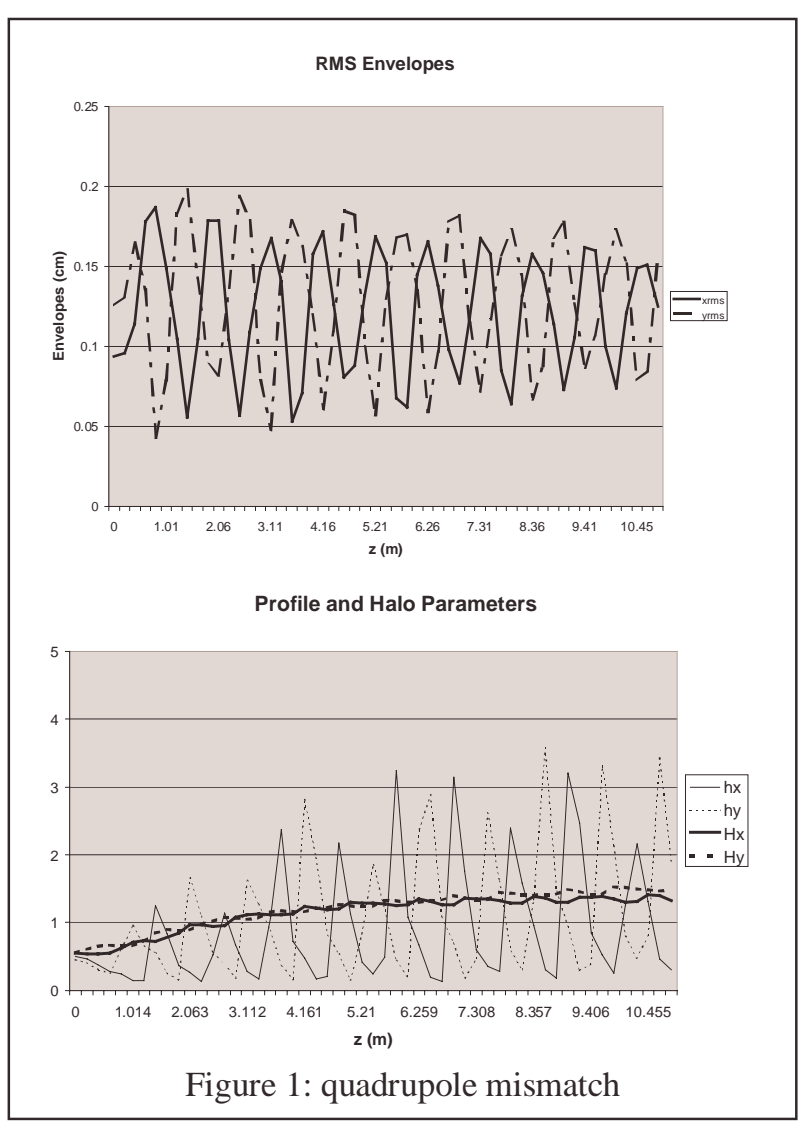

\section{CONCLUSION}

The halo parameters defined here represent a convenient and model-independent method for quantifying the magnitude of beam halo observed in phase space and spatial projections. The profile parameter $h$ is, in essence, the kurtosis of the beam distribution. The phase-space halo parameter $H$ can be interpreted as a generalization of $h$ into 2D phase space such that it is invariant under linear forces. Both parameters are useful. Although the profile parameter and halo parameter both reduce to the same value when the distribution has the elliptically symmetric form of Eq. (4), in general these parameters are not equal.

\section{Acknowledgements}

We wish to acknowledge Nicholas Pichoff for the suggestion of using kinematic invariants.

\section{REFERENCES}

[1] A.J. Dragt, R.L. Gluckstern, F. Neri, and G. Rangarajan, Frontiers of Particle Beams; Observation, Diagnosis and Correction, Lecture Notes in Physics 343, edited by M. Month and S. Turner (Springer-Verlag, Berlin, 1988), pp. 94-121.

[2] D.D. Holm, W.P. Lysenko, and J.C. Scovel, J. Math. Phys., Vol. 31, No. 7 (July, 1990), pp. 1610-1615.

[3] T.P. Wangler and K.R. Crandall, "Beam Halo in Proton Linac Beams", Linac 2000 Conference Proceedings, Monterey California, August 21-25, 2000. 